\title{
Integrated Mode-Locked Lasers in a CMOS-Compatible Silicon Photonic Platform
}

\author{
Cheryl Sorace-Agaskar ${ }^{1}$, Patrick T. Callahan ${ }^{1}$, Katia Shtyrkova $^{1}$, Anna Baldycheva ${ }^{1,5}$, Michele Moresco ${ }^{1}$, \\ Jonathan Bradley ${ }^{1}$, Michael Y. Peng ${ }^{1}$, Nanxi Li ${ }^{1}$, E. Salih Magden ${ }^{1}$, Purnawirman ${ }^{1}$, Michelle Y. Sander ${ }^{1,6}$, \\ Gerald Leake $^{2}$, Douglas D. Coolbaugh ${ }^{2}$, Michael R. Watts ${ }^{1}$, and Franz Kärtner ${ }^{1,3,4}$ \\ ${ }^{1}$ Massachusetts Institute of Technology, Research Laboratory of Electronics, 50 Vassar St. Cambridge, MA 02139, USA \\ ${ }^{2}$ College of Nanoscale Science and Engineering, University at Albany, 1400 Washington Ave., Albany NY, 12222, USA \\ ${ }^{3}$ Department of Physics, University of Hamburg, Notkestraße 85, Hamburg 22607, Germany \\ ${ }^{4}$ Center for Free-Electron Laser Science, Deutsches Elektron-Synchrotron, Luruper Chaussee 149, Hamburg 22761, Germany \\ ${ }^{5}$ University of Exeter, Harrison Building, North Park Road, EX4 4QF, Exeter, UK \\ ${ }^{6}$ Department of Electrical and Computer Engineering, Boston University, Boston, MA 02215 \\ Authore-mail address: ch20117@mit.edu
}

\begin{abstract}
Integrated components necessary for a mode-locked laser are demonstrated on a platform that allows for monolithic integration with active silicon photonics and CMOS circuitry. CW lasing and Q-switched mode-locking are observed in the full structures.

OCIS codes: (140.4050) Mode-locked lasers; (130.3120) Integrated optics devices.
\end{abstract}

\section{Introduction}

Mode-locked lasers (MLLs) are important for many applications. In particular, the low timing jitter between pulses makes them attractive for low-noise microwave generation, optical sampling and for frequency combs. As silicon photonics matures to the point where full systems can be implemented on-chip, the ability to include a MLL allows for the possibility of very compact size, weight and power systems that take advantage of these properties.

Previous demonstrations have shown MLLs integrated on-chip using erbium-doped alumino-silicate on a planar silica waveguide platform [1]. However, this system cannot be directly integrated with active silicon photonics or CMOS for further data processing. Additionally, monolithic integration is desired because it would allow for cheap and fast mass production of desired systems. Integrated MLLs have also been shown in III-V materials on silicon [2]. While this allows for bonding to active photonic and CMOS circuitry, the noise properties of III-Vs ultimately limit the performance of the MLL to levels below that achievable in solid-state MLLs.

In our group we have been demonstrating an integrated platform that allows for solid-state erbium-based lasers with low-noise characteristics to be integrated with active silicon photonics and CMOS electronics [3]. This paper will show progress toward including a mode-locked laser in this platform.

\section{Platform and Components}

The silicon photonic, CMOS, and laser layer stack is shown in Fig. 1.b. The CMOS and Photonics wafers are fabricated in a 300-mm CMOS foundry. The laser waveguide is comprised of a patterned nitride layer to guide the light and an etched trench in which an erbium-doped $\mathrm{Al}_{2} \mathrm{O}_{3}$ gain medium is deposited (Fig.1.). To prevent the laser mode from being strongly confined in the nitride and not interacting with the gain layer, the nitride layer is cut into five pieces to lower the effective index (Fig.2.a.). The waveguide has a single TE mode guided by the nitride ridge in which laser oscillation occurs, but the waveguide structure also supports multiple unwanted modes guided by the trench walls. To attenuate these modes so they do not compete for gain, the sides of the trench wall are roughened. Fig. 2.a. shows the cross section of the gain waveguide including the roughened area. Transmission measurements of the waveguide without roughing clearly show multimode beating; those of the roughened waveguide show that this beating has been removed without adding significant propagation loss. The net waveguide gain at $1550 \mathrm{~nm}$ was measured to be $0.5 \mathrm{~dB} / \mathrm{cm}$. Outside the laser trench a second nitride layer is present to help guide the light.
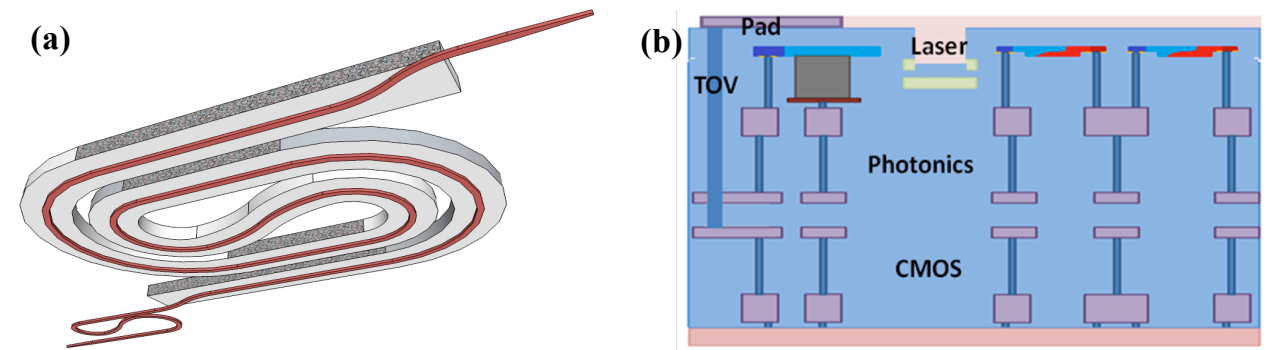

Fig. 1. a. Diagram of integrated spiral laser, b. Diagram of integrated photonics platform.

An integrated loop mirror (Fig 2.c.) made in this double layer of nitride serves as an output coupler. This mirror allows for the necessary reflections in the desired band around $1550 \mathrm{~nm}$, while transmitting the $1480 \mathrm{~nm}$ pump light into the laser cavity. The loop mirror and trench transition are both polarization sensitive, and their combined loss 
for the TM mode suffices to ensure that oscillation only occurs in the TE mode. On the other end of the laser, a taper serves to take the light to the edge of the chip where it is reflected off a butt-coupled saturable absorber to allow passive mode-locking. The losses from this taper were measured to be $<2 \mathrm{~dB}$ for each round trip reflection.

(a)
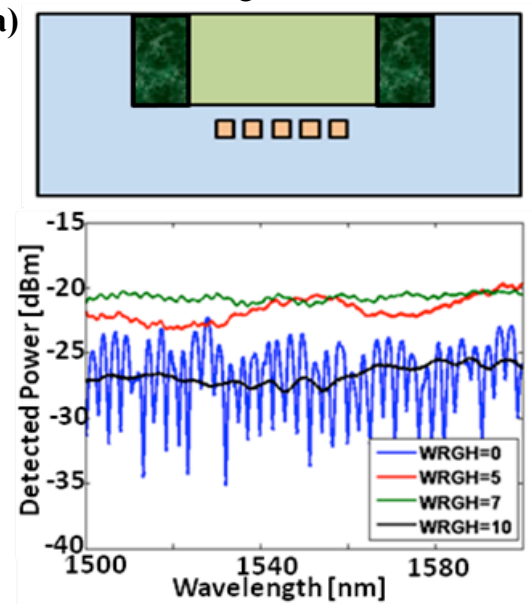

(b)
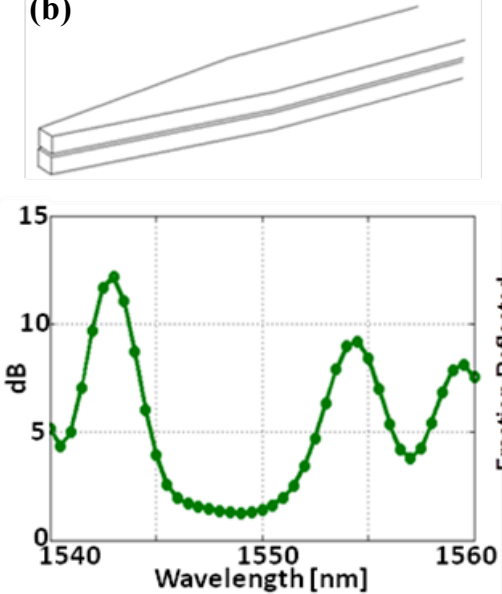

(c)
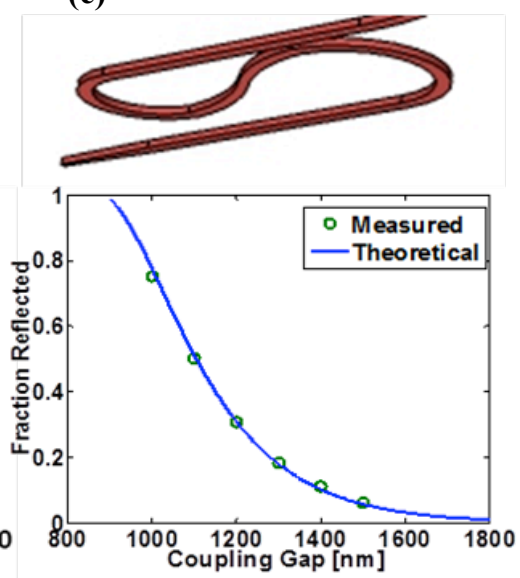

Fig.2.(a). Schematic cross section of laser waveguide with sidewall roughness and measurement results. Multimode beating is clearly seen for the case of no roughness (blue line), a roughness width of 5 or $7 \mu \mathrm{m}$ removes unwanted modes (as seen by lack of beating), but does not add to loss (green and red line), a roughness of $10 \mu \mathrm{m}$ causes extra loss (black line), (b). Taper off chip to saturable absorber showing loss as a function of wavelength for round-trip reflection. Loss on the order of 1-2 dB is achieved for the wavelength band of interest around 1550nm, (c). Integrated

\section{Lasing results} nitride loop mirror, showing good match between theory and experiment at $1550 \mathrm{~nm}$.

The full laser architecture is shown in Fig1.a. It is comprised of a $20 \mathrm{~cm}$ spiral of gain waveguide with the loop mirror output coupler on one side and the taper to a semiconductor saturable absorber on the other. The laser was tested in continuous-wave $(\mathrm{CW})$ operation by butt-coupling a mirror to the chip facet. The spectrum of the resulting $\mathrm{CW}$ laser is shown in Fig.3.a. Fig3.b. shows the laser output power as the pump power is increased, demonstrating operation above threshold.

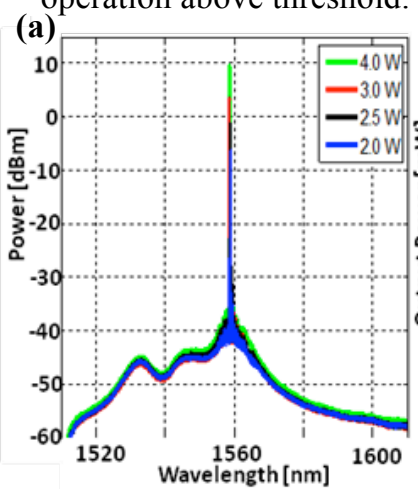

(b)

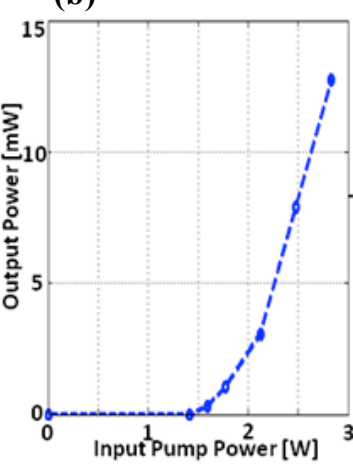

(c)

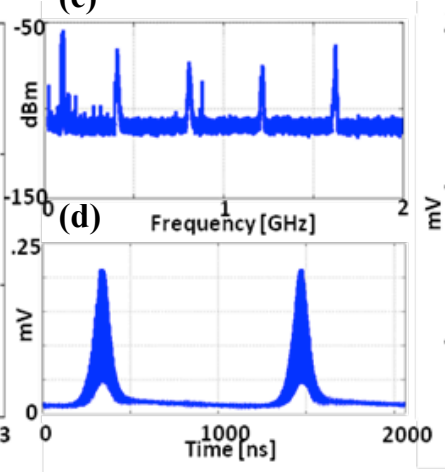

(e)

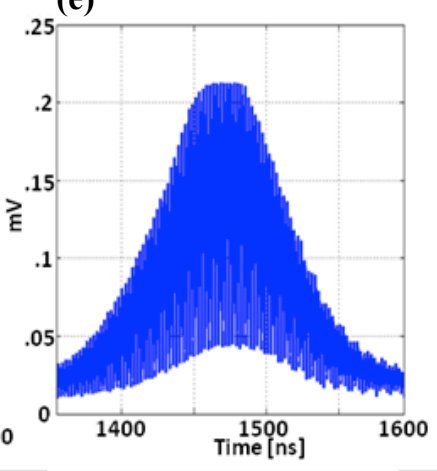

Fig. 3. a. Cw laser output spectrum vs. pump power, b. Laser output power vs. input pump power on chip, c. RF spectrum of laser in Q-switched mode-locking regime, $d$. oscilloscope trace of same, e. Zoomed in image of pulse burst.

Next, the mirror was exchanged for a semiconductor saturable absorber mirror and the laser was retested. The oscilloscope trace and resulting RF spectrum are shown in Fig.3.c. The expected $\sim 400 \mathrm{MHz}$ repetition rate is clearly seen on the RF spectrum analyzer. However, the comb line amplitudes are highly variable. This is due to the additional presence of Q-switching in the cavity as can be seen from the oscilloscope trace. Presence of Q-swtiched mode-locking is verified by the fact that each pulse burst consists of pulses spaced by the MLL repetition rate. We suspect that this Q-switched mode-locking is caused by an excess of normal dispersion in the cavity and are currently redesigning the components in order to achieve net anamalous dispersion and cw-mode-locking. In conclusion, components and preliminary operation of an integrated MLL that can be fabricated in a silicon photonics + CMOS platform were demonstrated.

\section{Acknowledgments}

This work was supported under the DARPA E-PHI project, grant no. HR0011-12-2-0007.

\section{References}

[1] H. Byun, et al. "Integrated Low-Jitter 400-MHz femtosecond waveguide laser," IEEE PTL, vol. 21. no. 12. pp 763-765. (2009)

[2] B.R. Koch, et al. "Mode-locked silicon evanescent lasers," Optics Express, vol. 15, no. 18. pp.11225-11233 (2007)

[3] M.R. Watts, et. al. "An Electronic-Photonic 3D Integration Platform,” GORMAC, Las Vegas, NV, USA, (2013) 